\title{
Optimization of 3D Triangular Mesh Watermarking Using ACO-Weber's Law
}

\author{
Modigari Narendra $^{1^{*}}$, Dr. M.L.Valarmathi ${ }^{2}$, Dr. L.Jani Anbarasi ${ }^{3}$ \\ ${ }^{1}$ Assistant Professor, Computer Science and Engineering , Vignan’s Foundation for Science \\ Technology and Research Deemed to be University,Guntur \\ [e-mail: narendramodigari@gmail.com] \\ ${ }^{2}$ Professor, Computer Science \& Engineering, Dr. Mahalingam College of Engineering and Technology, \\ Pollachi, India \\ [e-mail: drmlv@gct.ac.in] \\ ${ }^{3}$ Assistant Professor(Sr), School of Computing Science and Engineering,VIT Chennai \\ [e-mail: jani_lj_2000@yahoo.com] \\ *Corresponding author : Modigari Narendra
}

Received December 21, 2019; revised August 17, 2020; accepted September 15, 2020; published October 31, 2020

\begin{abstract}
The development of new multimedia techniques such as 3D printing is increasingly attracting the public's attention towards 3D objects. An optimized robust and imperceptible watermarking method based on Ant Colony Optimization (ACO) and Weber Law is proposed for 3D polygonal models. The proposed approach partitions the host model into smaller sub meshes and generates a secret watermark from the sub meshes using Weber Law. ACO based optimized strength factor is identified for embedding the watermark. The secret watermark is embedded and extracted on the wavelet domain. The proposed scheme is robust against geometric and photometric attacks that overcomes the synchronization problem and authenticates the secret watermark from the distorted models. The primary characteristic of the proposed system is the flexibility achieved in data embedding capacity due to the optimized strength factor. Extensive simulation results shows enhanced performance of the recommended framework and robustness towards the most common attacks like geometric transformations, noise, cropping, mesh smoothening, and the combination of such attacks.
\end{abstract}

Keywords: Ant colony optimization, Digital Watermarking, 3D models, Weber's Law, Image Processing 


\section{Introduction}

Copyright protection of digital multimedia models [1] has been the focal point in multimedia research for a long period. In the field of multimedia and computer graphics, importance towards copyright protection is rapidly growing to overcome the unauthorized duplication. The progress of internet and digital signal processing have made copying, transferring, conversion and sharing of multimedia documents so simple. Watermarking scheme is a method that embeds hidden information called watermark into the host, which holds the exact owner information. Whenever the ownership of any multimedia object is under question the secret watermark is extracted to make a claim about the object. Watermarking schemes can be used for various applications like transaction tracing, copyright and copy protection, broadcast monitoring, medical safety and authentication etc., [2-4].

Watermarking techniques can be categorized as i) fragile and ii) robust. Fragile watermarking authenticates the digital content and is able to detect even the smallest changes that have occurred to the system. Robust watermarking [33, 34] hides a simple logo or a secret into the digital content to ensure the ownership details. This embedded secret should be robust against intentional or unintentional attacks and should be able to extract the secret without loss. Similarly watermarking is also classified as blind, non-blind watermarking. For non-blind watermarking schemes, the original data is required during the extraction process. This scheme achieves good imperceptibility, high robustness towards various attacks and has a limited utilization effect. In contrast, blind mesh watermarking doesn't require the original secret during the extraction process[26]. Most of the blind watermarking schemes are mostly vulnerable to cropping attacks[29]. Watermarking in the 3D mesh models can be categorized according to the domain in which the watermarks are embedded or extracted. In accordance with this, watermarking schemes are classified as spatial and spectral domain techniques. In spatial domains, the watermarks are embedded [28] through modifying the structure of the mesh line vertex coordinates, edge length, triangular area, tetrahedral volume, etc. Generally, spatial schemes are designed to be in both fragile and robust, but we have to perform some additional operations to increase its robustness. A spectral method embeds the secret watermark in the coefficients of harmonic coefficients or transform domain like wavelet or frequency.

Similarly watermarking can be classified as blind, non-blind, and semi-blind [35, 36]. Blind watermarking schemes allow the authorized user to extract the watermark using the secret key. It doesn't require either the original content or the watermarked content [27]. Non-blind watermarking schemes require the original data and the watermark to verify the watermarks embedded in the applications. The original data is not required in the semi-blind watermarking scheme. A novel blind watermarking is proposed by [37, 38] using DWT and DCT for images to obtain strength of imperceptibility and robustness. The watermark is embedded into the joined transform to overcome the individual drawbacks and results in an effective watermarking. Similarly the watermarking is performed to embed the watermark in the special middle frequency. Similarly to optimize the watermarking the various research work has been performed. Robust and novel insertion using discrete wavelet transform and ANN was performed to insert based on the association between the coefficient and its neighbors' standard deviation. Vertices for embedding the watermark are selected by back propagation neural network [39]. 
This paper proposed an approach to embed the watermark by modifying the wavelet coefficient of the 3D models. The secret watermark is generated from the actual 3D model using the Weber law. Vertices of the 3D models for embedding is selected using the optimized strength factor computed using ACO. Watermark is extracted from the original 3D model in the presence of watermarked model. This scheme enhances the robustness and imperceptibility and is compared with the existing algorithm. The remainder of this paper is structured as follows. Section 2 describes the related works. The proposed study is explained in Section 3. This section explains in detail about the proposed pre-processing, feature extraction and classification methods used for classifying the COVID-19 Lung X-ray images. Section 4 illustrates the experimental analysis, results and discussion followed by Conclusions in Section 5.

\section{Related Work}

3D digital watermarking schemes are relatively new compared to 2D watermarking. 3D models has received less contribution as the techniques implemented for video content and image data cannot be adapted as such due to its complex representation like voxels, NURBS, polygonal meshes etc. Benedens [6] proposed geometry based watermarking which directly embedded the watermark by altering the 3D mesh geometry. Similarly Ohbuchi [7] and Harte [8] also embedded the secret watermark by altering the geometry and topology of the 3D mesh models. These schemes are simple which requires less computation and are not robust enough to resist various attacks.

Later various investigations were performed in frequency domain based on $[5,7,11]$ spectral decomposition and wavelet transformation, which achieved a good resistance towards various attacks. Praun [5] proposed a generalized spread spectrum technique which constructed a scalar basic function using multi resolution technique over the vertices of the mesh model. The secret is embedded into the vertices of the 3D model along the surface weighted by a basic function. Obhuche [7] proposed a mesh spectral matrix to embed the secret watermark into the spectral coefficient. Cotting et al [9] generalized the mesh spectral coefficient to point based embedding in 3D geometrics. Qiu et al [11] decomposed the original 3D model into a various series of details using spherical wavelet transform and embedded the secret watermark in the approximation part of the transformed 3D model. Li et al [10] embedded the watermark into partitioned surfaces using additive characteristic of Fourier transform. Kwon et. al.[12] watermarked into every polygon patch using normal vector distribution. A blind robust watermarking technique is analyzed by Zafeiriou et. al. [16] for copyright protection which is robust enough to resist against affine transformation attacks. A new texture based 3D video object watermarking was suggested by Garcia et al [15] and Ucceddu et. al. [14] where the watermark is embedded at suitable resolution level after performing wavelet decomposition. A new orthogonal function based on radial basis was proposed by [13] which generated a robust spectral scheme for large meshes. Karni and Gotsman [22] performed a mesh Laplacian matrix to encode 3D shape using Eigen values which successfully retained the shape information without loss. A new symmetric encryption scheme is proposed based on Arnonld cat map, using shuffling and substitution. A novel robust statistical watermarking of 3D mesh was performed by Nassima et al [6], embedded the signature bits into the vertices identified through a salient point detector [1] ADF (Auto Diffusion Function). The bits are embedded repeatedly into each region thus overcoming the cropping attack. The authors further extended by segmenting the mesh based on the 
identified salient features. Statistical embedding of secret was performed by altering the amplitude and means of the bits watermark strength [3]. Han et. al.[4] presented an embedding approach through consistent segmentation and steganalysis. The primary watermark domain is identified using the shape function and the consistency interval is used to eliminate the outliers of the segments. This scheme also followed the principle of embedding the same watermark into various segments thus achieving high robustness against the cropping attack.

\section{Representation of Triangle Mesh and Methodologies}

3D object are typically characterized as triangular or polygonal meshes in graphics and geometric-aided designs. The triangular meshes are well-described as $\mathrm{M}=(\delta, \beta)$ where $\delta=$ $\left(\delta_{1}, \delta_{2}, \delta_{3} \ldots \delta_{\mathrm{m}}\right)$ is the set of vertices and $\beta=\left(\beta_{1}, \beta_{2}, \ldots \beta_{\mathrm{n}}\right)$ is the set of faces of the 3D model. Every edge $\mathrm{l}_{\mathrm{ij}}=\left(\delta_{\mathrm{i}}, \delta_{\mathrm{j}}\right)$ is formed by connecting vertex pairs where $\delta_{\mathrm{i}}, \delta_{\mathrm{j}} \in \delta$ are said to be adjacent if their edges are connected i.e. $\mathrm{e}_{\mathrm{ij}} \in \beta$.

\section{A. Weber Law}

Weber Law [20, 21] demonstrates the relation between incremental threshold and intensity as constant. Relationship between the intensity and quantity in Weber's Law is termed as

$$
\frac{\partial T}{T}=C
$$

Where incremental threshold is $\partial T$, primary value of intensity is $T$ and fractional constant is $C$. For any vertex $\delta$ the coordinates is chosen from $\mathrm{x}, \mathrm{y}$ and $\mathrm{z}$ axes of mesh models where $\delta$ represents the center vertices surrounded by eight neighbors which are shown in Fig. 1.

\begin{tabular}{|l|l|l|}
\hline$\delta_{1}$ & $\delta_{8}$ & $\delta_{7}$ \\
\hline$\delta_{2}$ & $\delta_{9}$ & $\delta_{6}$ \\
\hline$\delta_{3}$ & $\delta_{4}$ & $\delta_{5}$ \\
\hline
\end{tabular}

Fig. 1. Representation of Vertex Neighborhood

Weber Law organizes the data into non overlapping block from which the threshold intensity among neighbors of every pixel is assumed as the differential excitation descriptor. Differential excitation $\chi$ is calculated for the current vertex $\delta$ as (2), where $m$ lies between 1 to 8 .

$$
\chi\left(\mathrm{x}_{\mathrm{i}}, \mathrm{y}_{\mathrm{j}}\right)=\arctan \left(\sum_{\mathrm{n}=0}^{\mathrm{m}-1} \frac{\delta_{\mathrm{n}}-\delta_{\mathrm{i}}}{\delta_{\mathrm{i}}}\right)
$$

$\left(x_{i}, y_{j}\right)$ refers to the current vertices and ' $\mathrm{m}(=8)$ ' refers to the neighbors. The range of the differential excitation $\chi$ lies within the range $\left[\frac{\pi}{2}, \pi\right]$. The second descriptor of Weber's Law refers to the orientation $\lambda$ which is computed as follows

$$
\lambda\left(\mathrm{x}_{\mathrm{i}}, \mathrm{y}_{\mathrm{j}}\right)=\arctan \left(\frac{\delta_{8}-\delta_{4}}{\delta_{6}-\delta_{2}}\right)
$$


Orientation $\lambda$, is mapped to $\lambda_{2}$ using Eq.(4), such that $\lambda_{2}$ lies in between the interval [0, $2 \pi]$.

$$
\lambda_{2}\left(\mathrm{x}_{\mathrm{i}}, \mathrm{y}_{\mathrm{j}}\right)=\arctan 2\left(\frac{\delta_{7}-\delta_{3}}{\delta_{5}-\delta_{1}}\right)+\Pi
$$

$\lambda_{2}$ refers to the orientation of the processed vertices $\left(\mathrm{x}_{\mathrm{i}}, \mathrm{y}_{\mathrm{j}}\right)$.

\section{B. Ant Colony Optimization (ACO)}

Ant Colony Optimization is a meta heuristic algorithm introduced by Dorigo [17, 18, 32] to resolve complex optimization issues. ACO algorithm is enlivened by the behavior of ant's species during their exploration of food surrounding their nest [19]. If the ants identify their food, then they drop pheromone a chemical substance on the pathway to trace the food source. All the other ants smell the trace through high concentration of pheromone. Thus optimal path to the food source and the nest is found through the pheromone, which helps in effective transfer of food to their nest. The representation of colony within ACO algorithm is the initial process. A matrix $\mathrm{T}$ of size $M x N$ represents the colony of $M$ ants where $T=\left(y_{1}, y_{2} \ldots . . y_{m}\right)$ and $Y=\left(y_{1}, y_{2}, y_{3}, \ldots \ldots . y_{n}\right)^{t}$ corresponds to vectors of $\mathrm{N}$ variables of a single ant. Socha and Dorigo [36] computed $\eta_{\text {phoromone }}$

$$
\eta_{\text {phoromone }}=\exp \left(-\frac{\left(y-y_{\min }\right)^{2}}{2 \sigma^{2}}\right)
$$

through normal distribution given in equation 5 where $y_{\text {min }}$ refers the optimal point identified from the design space. Standard deviation $\sigma$ specifies the current minimum value through aggregating the ants $y_{\min }$ and $\sigma$ are initialized accordingly. $y_{\min }$ is initialized randomly through uniform distribution computation and $\sigma$ is chosen greater than the span of the design space so that the it can be located inside. Each design variables of every ant are updated after every iteration and the distribution of pheromone is updated using the data obtained through the optimization as given in the following equation 6

$$
\sigma=\operatorname{std}(\text { antcolony })
$$

where std(antcolony) [32] is the elite solution which retrieves a vector that includes the standard derivation for every design variable. Ideal solution can be obtained by accumulation of Pheromone through increase in the vicinity of the candidate. Negative updating processes are not disregarded to prevent premature convergence. So a modest scheme to dissolve the pheromone is performed where the quantity of pheromone is modified by the current standard deviation of each variable as $\sigma_{\text {new }}=\lambda . \sigma_{\text {old }}$ where the dissolving rate is always considered as $(\lambda>1)$. 


\section{Proposed 3D Watermarking Scheme}

The watermarking scheme proposed by Laumar [21] and Anbarasi [20] using Weber's Law shows that the generated watermarked model is similar to the host 3D model for strength factor $\alpha$ equal to 0 , whereas the retrieved watermark will be close to original watermark for strength factor $\alpha$ equal to 1 . Identifying a strength factor to generate a less visually degraded watermarked model with a good retrieval property was performed using a trial and error method in these schemes. The analyses were performed with a strength factor ranging from $\alpha=0.98$ to 0.001 and the identified value doesn't achieve good imperceptibility all the times due to the complex nature of the mesh model.

Mostly high scalar value affects the imperceptibility but improves robustness. Challenging task deals with the computation of optimal strength factor to achieve high imperceptibility and robustness. Near optimum strength factor has to be identified for good visual quality and robustness. Optimization algorithm plays an inevitable role in identifying the optimum strength factor. So an optimized strength factor using ACO for watermarking in 3D models is suggested and analyzed in this work.

In this proposed method, a 3D model fingerprint information is framed from the host 3D model based on Weber differential excitation descriptor $\chi$. The 3D mesh model is partitioned into $3 \times 3$ sub mesh models and for every $3 \times 3$ non overlapping sub model, differential excitation descriptor value is computed to form vertices of the watermark. The faces of the host watermark are included in the vertices thus constructing a fingerprint watermark. This fingerprint watermark is embedded into the host 3D model for an elite strength factor. The generation of the fingerprint secret watermark, embedding and retrieval is expressed in the following section. The proposed optimized watermarking architecture for 3D models is shown in Fig. 2.

\section{A. Generation of Watermark}

Every three vertex coordinates are grouped into $3 \times 3$ blocks, resulting in ' $\mathrm{c}$ ' generating blocks. The Weber differential excitation description value $\chi$ is computed based on the threshold intensity among the neighbours of each generating block as expressed in the equation 7.

$$
W\left(\mathrm{x}_{\mathrm{i}}, \mathrm{y}_{\mathrm{j}}\right)=\sum_{\mathrm{x}=1}^{\mathrm{x}=\mathrm{m} / 3} \sum_{\mathrm{y}=1}^{\mathrm{y}=3} \arctan \left(\sum_{\mathrm{n}=0}^{\mathrm{m}-1} \frac{\delta_{\mathrm{n}}-\delta_{\mathrm{i}}}{\delta_{\mathrm{i}}}\right)
$$

' $\mathrm{c}$ ' generating blocks results in ' $\mathrm{c}$ ' values and every 3 coordinates are grouped to form vertex values as $\delta_{\mathrm{x}}, \delta_{\mathrm{y}}, \delta_{\mathrm{z}}$. Based on the number of vertices, faces are included from the host model to generate a fingerprint 3D watermark and the resulted secret watermarks for various models are given in Table 2.

\section{B. Embedding of Watermark}

The fingerprint 3D model is embedded in the ' $\mathrm{C}$ ' generating blocks of host 3D model using linear interpolation given as equation (8)

$$
W_{m}=\sum_{x=1}^{x=m / 3} \sum_{y=1}^{y=3}(1-\alpha) * w_{c}+\alpha * i_{c}
$$

Where $\mathrm{W}_{\mathrm{m}^{-}}>$watermarked block, $\mathrm{w}_{\mathrm{c}^{-}}>$watermark vertices (coordinates of the block $\mathrm{c}$ ), $\mathrm{i}_{\mathrm{c}^{-}}{ }^{->}$ center value of the host 3D model, $\mathrm{m}->$ maximum vertex. 


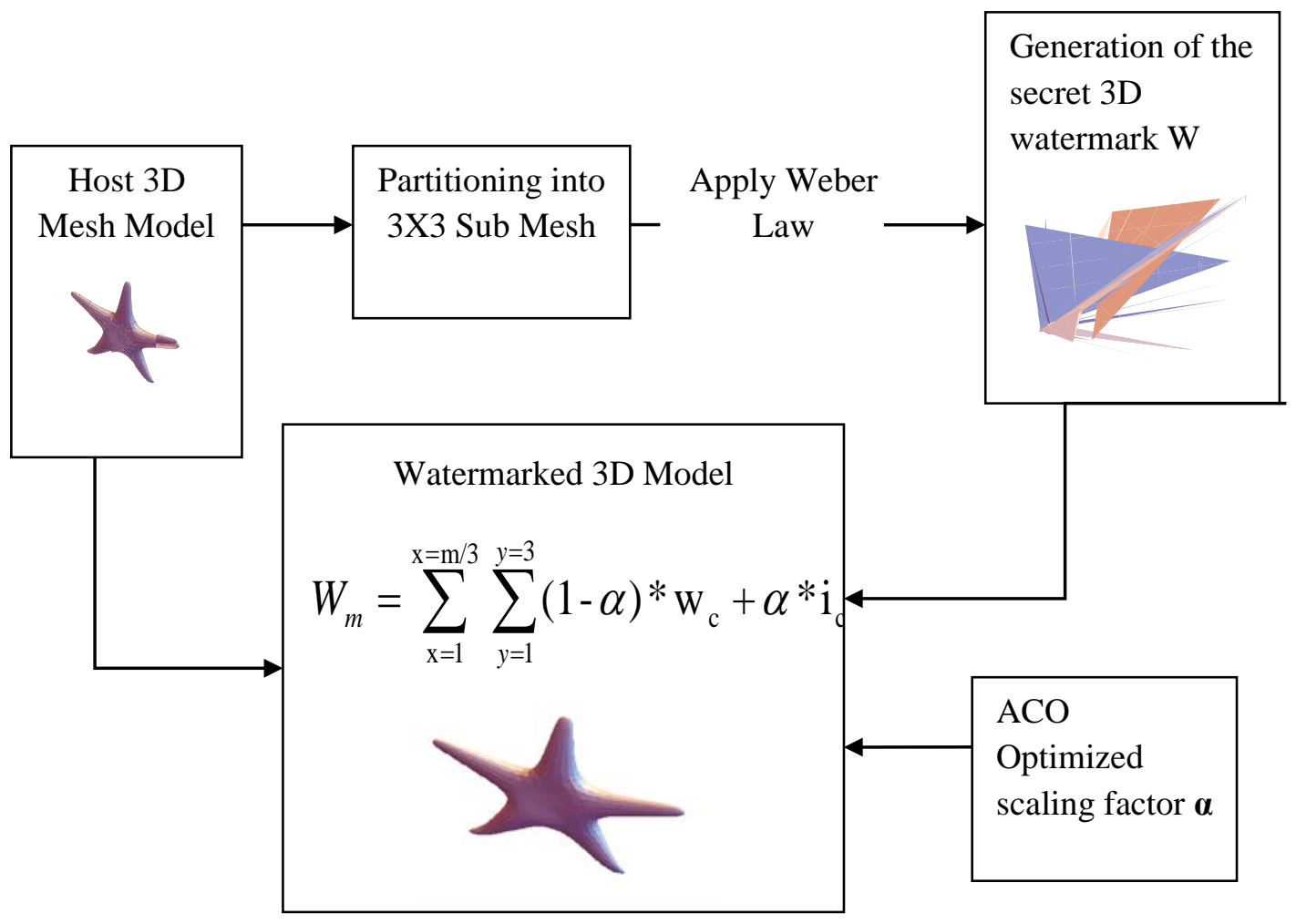

Fig. 2. Architecture of the Proposed System

\section{Watermark Extraction}

The proposed semi blind watermarking scheme extracts the watermark, by performing inverse linear interpolation on the watermarked 3D model, splitting into ' $C$ ' blocks of size $3^{*} 3$ as given in equation (9) Where $\mathrm{W}_{\mathrm{e}}$ is the extracted watermark, $\alpha$ is the optimized strength factor

$$
W_{e}=\sum_{x=1}^{x=m / 3} \sum_{y=1}^{y=3} \frac{1}{\alpha} W-\frac{(1-\alpha)}{\alpha} W_{m}
$$

\section{Identification of Optimized strength factor}

The algorithm for identifying the optimized strength factor $\alpha$ is given as

a) Initialize the parameters of ACO such as population, fitness function and stopping criterion.

b) Target fitness function is defined as a good PSNR value $\geq 50 \mathrm{~dB}$ and a high NC $(\mathrm{NC}=1)$.

c) Randomize the Initial population of ants $\mathrm{X}$ which includes a set of potential solution to that in equation 5 .

d) For each ant termed as $\mathrm{X}$ of length $\mathrm{L}$ in population perform the following:

1. Embed the watermark using $X$ as strength factor as given in equation 8 .

2. Compute NC between host (I) and watermarked model ( $\left.\mathrm{I}_{\mathrm{m}}\right)$ 
3. For each watermarked model perform few attacks like cropping, scaling and noise addition, generate different attacked watermarked model as $W_{c}, W_{s}, W_{r} € \mathrm{~W}_{\mathrm{i}}$.

4. Extract the watermark from the attacked model $\hat{W}_{i}$

5. Compute $\mathrm{NC}$ between watermark and the retrieved secret watermark $\left(\mathrm{w}, \hat{\mathrm{w}}_{\mathrm{m}}\right)\left(\mathrm{w}, \hat{\mathrm{w}}_{\mathrm{c}}\right)\left(\mathrm{w}, \hat{\mathrm{w}}_{\mathrm{s}}\right)\left(\mathrm{w}, \hat{\mathrm{w}}_{r}\right)$

$\hat{\mathrm{W}}_{\mathrm{m}}$ - Secret watermark retrieved from original watermarked model,

$\hat{\mathrm{w}}_{\mathrm{c}}$ - Cropped model, $\hat{\mathrm{w}}_{\mathrm{s}}$ - Scaled, $\hat{\mathrm{w}}_{\mathrm{r}}$ - Noisy model

6. Fitness function is computed as (10)

$$
F(x)=\left(\frac{1}{N C\left(I, I_{m}\right) N C\left(w, w_{m}\right) N C\left(w, \hat{w}_{c}\right) N C\left(w, \hat{w}_{s}\right) N C\left(w, \hat{w}_{r}\right)}\right)
$$

7. Elite strength value is computed using multi objective function as given in equation (11) where constant values are $\mathrm{p}, \mathrm{w}, \mathrm{F}_{0}$

$$
f_{o b j}(x)=\sum_{i=1}^{T+2}\left(e^{p . w}-1\right) e^{p\left(F(x)-F_{0}\right)}
$$

8. Pheromone is updated using equation (12) where $\sigma$ specifies finally evaluated value and their aggregation, where $x_{i}$ refers the present value in population matrix , $\hat{x}$ refers mean and ' $m$ ' is the population count .

$$
\sigma_{1}=\sqrt{\frac{1}{m}} \sum_{i=1}^{m} x_{i}-\hat{x}
$$

The maximum iteration is initialized to 150 and the target strength factor $\alpha$ is set as [0.98 to 0.01 ] and the value of $\mathrm{p}, \mathrm{w}, \mathrm{f}_{\mathrm{o}}$ is considered as 2,5 and 10 for equation (11).

e) If the maximum number of population or target objective function is reached (which is the stopping criteria) then goto step ' $\mathrm{f}$ ' or goto step ' $\mathrm{c}$ '.

f) The $\chi_{\text {best }}$ values with the smallest objective function $f_{o b j}$ are chosen as the strength factor to embed the watermark.

Table 1. Characteristics of the 3D host model and the secret 3D watermark

\begin{tabular}{|l|c|c|c|}
\hline Model & Vertex & Face & $\begin{array}{c}\text { Generated secret } \\
\text { Watermark (Vertices) }\end{array}$ \\
\hline Pinetree & 728 & 186 & 242 \\
\hline Piggybank & 4040 & 4008 & 1346 \\
\hline Golfball & 15560 & 16370 & 6058 \\
\hline Valcona & 12095 & 11796 & 4031 \\
\hline Vase & 4788 & 4707 & 1595 \\
\hline Potiron & 9736 & 10404 & 3244 \\
\hline Starfish & 1890 & 4070 & 629 \\
\hline
\end{tabular}




\begin{tabular}{|l|c|c|c|}
\hline $\begin{array}{l}\text { Wizards } \\
\text { Hat }\end{array}$ & 577 & 1080 & 191 \\
\hline Plane & 18176 & 35996 & 6058 \\
\hline
\end{tabular}

Table 2. Original 3D model and the Generated fingerprint watermarks

\begin{tabular}{|l|l|l|l|l|l|}
\hline \multirow{2}{*}{} & \multicolumn{5}{|c|}{ 3D Mesh Models } \\
\cline { 2 - 7 } & Starfish & Vase & Piggybank & Pinetree & Golfball \\
\hline $\begin{array}{l}\text { Host 3D } \\
\text { Model }\end{array}$ & & & & & \\
\hline $\begin{array}{l}\text { Generate } \\
\text { 3D } \\
\text { Watermar } \\
\mathrm{k}\end{array}$ & & & & & \\
\hline
\end{tabular}

Table 3. Watermarked 3D model, ACO strength factor and achieved PSNR

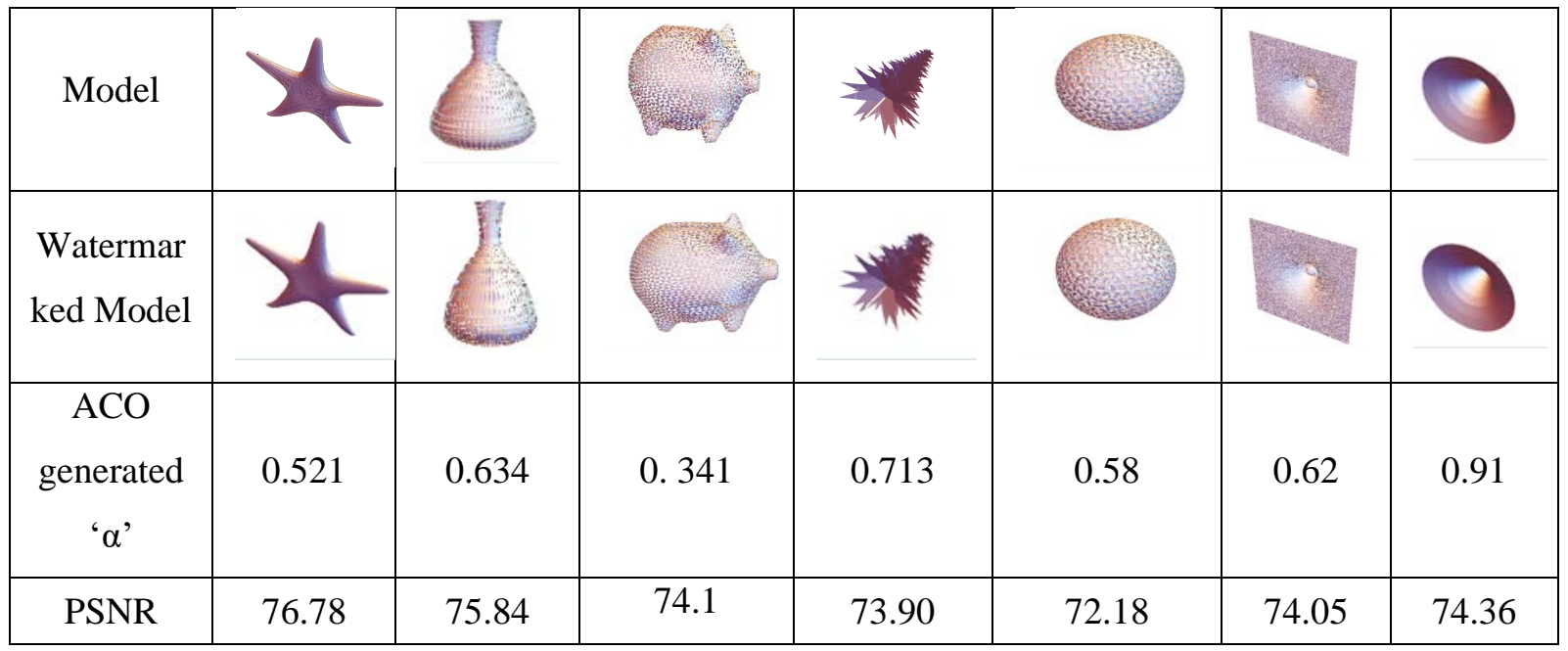

\section{Simulation Results and Evaluation}

The experimental evaluation of the proposed ACO-Weber watermarking scheme was analyzed with respect to imperceptibility (PSNR) and Robustness (NC) towards several known threats. The characteristics of 3D model (faces $F$, vertices $V$ ) used in the experimental study is given in Table 1. The optimized strength factor is obtained after several iteration of ACO algorithm. Table 3 presents the ACO based watermarked models 
and its obtained PSNR values. The results clearly predict a high imperceptibility in the resulting models. To assess the efficiency of the developed scheme, different simulation process was performed with various 3D mesh models.

\section{A. Mesh Distortion of ACO-Weber Law}

Degradation value of $3 \mathrm{D}$ model has to be measured to achieve a successful watermarking scheme. The mesh distortion of the watermarked models is measured using PSNR (Peak Signal to Noise Ratio) and NC (Normalized Correlation). PSNR measure is given in equation $(13)$ where $\max (\mathrm{I}(\mathrm{i}, \mathrm{j}))$ represents the vertex count and face coordinated of a 3D model.

$$
\text { PSNR }=10 \log _{10}\left(\max (I(\mathrm{i}, \mathrm{j}))^{2} / \mathrm{d}_{\mathrm{rms}}\right)
$$

Root mean square Error identifies the Geometrical Distortion between two meshes and is given in equation (14) where $\mathrm{M}$ and $\mathrm{M}^{\prime}$ refers to the original and deformed meshes surface and $\mathrm{v}$ refers to the point of the mesh model and $|\mathrm{M}|$ refers to the surface area of the 3D model $M$ and $d\left(v, M^{\prime}\right)$ refers to the point to mesh distance. This evaluates the geometrical distortion which is not symmetric [61].

$$
d_{r m s}\left(M, M^{\prime}\right)=\sqrt{d_{v \in M}\left(v, M^{\prime}\right)^{2} d M}
$$

Therefore the maximum root mean square error between a host and a watermarked model can be represented as equation 15. The distortions measured using MRMS is more accurate since it evaluates the mesh surface.

$$
d_{\text {rms }}\left(M, M^{\prime}\right)=\max \left(d_{r m s}\left(M, M^{\prime}\right), d_{r m s}\left(M^{\prime}, M\right)\right.
$$

Normalised Correlation NC is calculated as given in equation 16.

$$
\mathrm{NC}\left(\mathrm{w}, \mathrm{w}_{\mathrm{e}}\right)=\frac{\sum_{i=1}^{M} \sum_{j=1}^{N}\left(w(i, j)-\mu_{\omega}\right)\left(W_{e}(i, j)-\mu_{e}\right)}{\sqrt{\left.\sum_{i=1}^{M} \sum_{j=1}^{N}(w(i, j)-\mu \omega)^{2}\right)} \sqrt{\sum_{i=1}^{M} \sum_{j=1}^{N}\left(w(i, j)-\mu_{e}\right)^{2}}}
$$

$\mathrm{w}$ and $\mathrm{w}_{\mathrm{e}}$ represents the host and the extracted 3D watermark, $\mu_{\omega}, \mu_{e}$ indicates the mean value of original and the retrieved 3D secret watermark respectively. The normalized correlation value obtained for the original and extracted 3D mesh watermark lies in the range of -1 and 1 . The extracted 3D mesh watermark is identical to the original 3D mesh watermark while the Normalized Correlation value equals 1 . Fig. 3 shows the PSNR values obtained among the host and the watermarked 3D model compared to [20]. The results depicts that the optimized strength factor results in lesser degradation than a fixed strength factor.

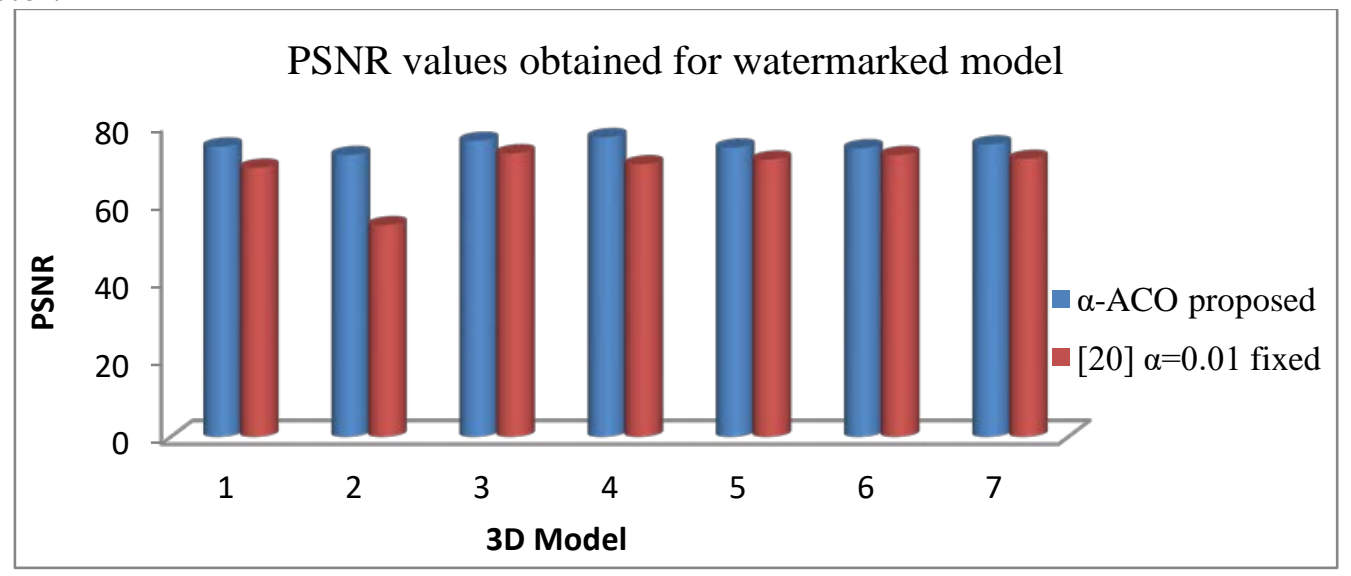

Fig. 3. Comparison of the PSNR values obtained among the host and the watermarked 3D model 
ACO based optimized Weber law resulted in optimized verifying strength factor for each model. The attained ideal strength factor successfully preserves a good imperceptibility PSNR values and good robustness against several attacks.

\section{B. Robustness of the proposed ACO-Weber watermarking Scheme}

To analyze the robustness of the proposed scheme, various attacks like geometrical transformation and topological transformation were tested. Geometrical transformation includes rotation, scaling, translation and their combinations, whereas topological transformations comprised attacks like cropping, rendering of vertices and mesh simplifications. Signal processing attack incorporates noising and compression. Table 4 shows the calculated results of the achieved robustness with respect to NC. The results shows that a high normalized correlation i.e. equivalent to the original $(\mathrm{NC}=1)$ is obtained for the proposed scheme.

Table 4. NC of the proposed ACO-Weber with respect to robustness

\begin{tabular}{|c|c|c|c|c|c|c|c|c|}
\hline \multirow{2}{*}{ Object } & \multicolumn{7}{|c|}{ Normalized Correlation values of different 3D model } \\
\cline { 2 - 8 } & Pinetree & Piggybank & Golfball & Valcona & Vase & Potiron & Starfish & $\begin{array}{c}\text { Wizard } \\
\text { s Hat }\end{array}$ \\
\hline $\begin{array}{c}\text { NC }(\alpha- \\
\text { ACO })\end{array}$ & 1.000 & 1.000 & 0.8948 & 1.000 & 0.997 & 1.000 & 1.000 & 1.000 \\
\hline $\begin{array}{c}{[21](\alpha} \\
=0.01 \\
\text { fixed })\end{array}$ & -0.6572 & 0.5121 & 0.8948 & 0.7907 & 0.332 & -0.3744 & -0.1462 & 0.8184 \\
\hline
\end{tabular}

Correlation is a suitable metric to compare and quantify the robustness to attacks. If the correlation of any watermark is achieved more than 0.5 then the watermark is visible. Three types of attacks are analyzed in our simulation studies: cropping, noising and scaling, various scenarios and different parameters used are specified in Table 5. Resistance towards noisy attack is shown by adding salt and pepper noise signal and Gaussian noise ranging from $0.5 \%, 1 \%, 2 \%$. The noisy 3D models along with the extracted watermarks are shown in Fig. 4(a, b). Fig. 5 shows the achieved correlation coefficient for different models. The results show a good NC for the retrieved watermark which is almost nearing 1. Similarly PSNR is achieved above $46 \mathrm{~dB}$ even after a noise ratio of $2 \%$ is added to the model.

Second the robustness towards cropping attack is tested for different degree of cropping vertices like $15 \%, 20 \%$, and $25 \%$. The secret watermark is extracted from the attacked model. Precisely in the case of cropping, most of the research works has embedded the secret in different region to resist cropping attack, but the proposed work achieves a correlation coefficient above 0.5 for lower strength factor thus overcoming the process of repetition to resist cropping attack. Fig. 6 details the obtained PSNR values of the retrieved watermark after cropping the vertices.

Similarly robustness against scaling attacks is analyzed using few factors a)scaling down $\mathrm{x}$ axis by $0.5 \%$ b) scaling up y-axis with $1 \%$ c)scaling along z-axis with $1.5 \%$ d) mixed scaling where $\mathrm{x}$-axis as $0.5 \%$, y-axis as $1 \%$ and z-axis as 1.5\%. Table 6 and Fig. 7 show the 
performance comparison between the proposed model and [20] to resist scaling attacks in terms of PSNR and NC detection ratio.

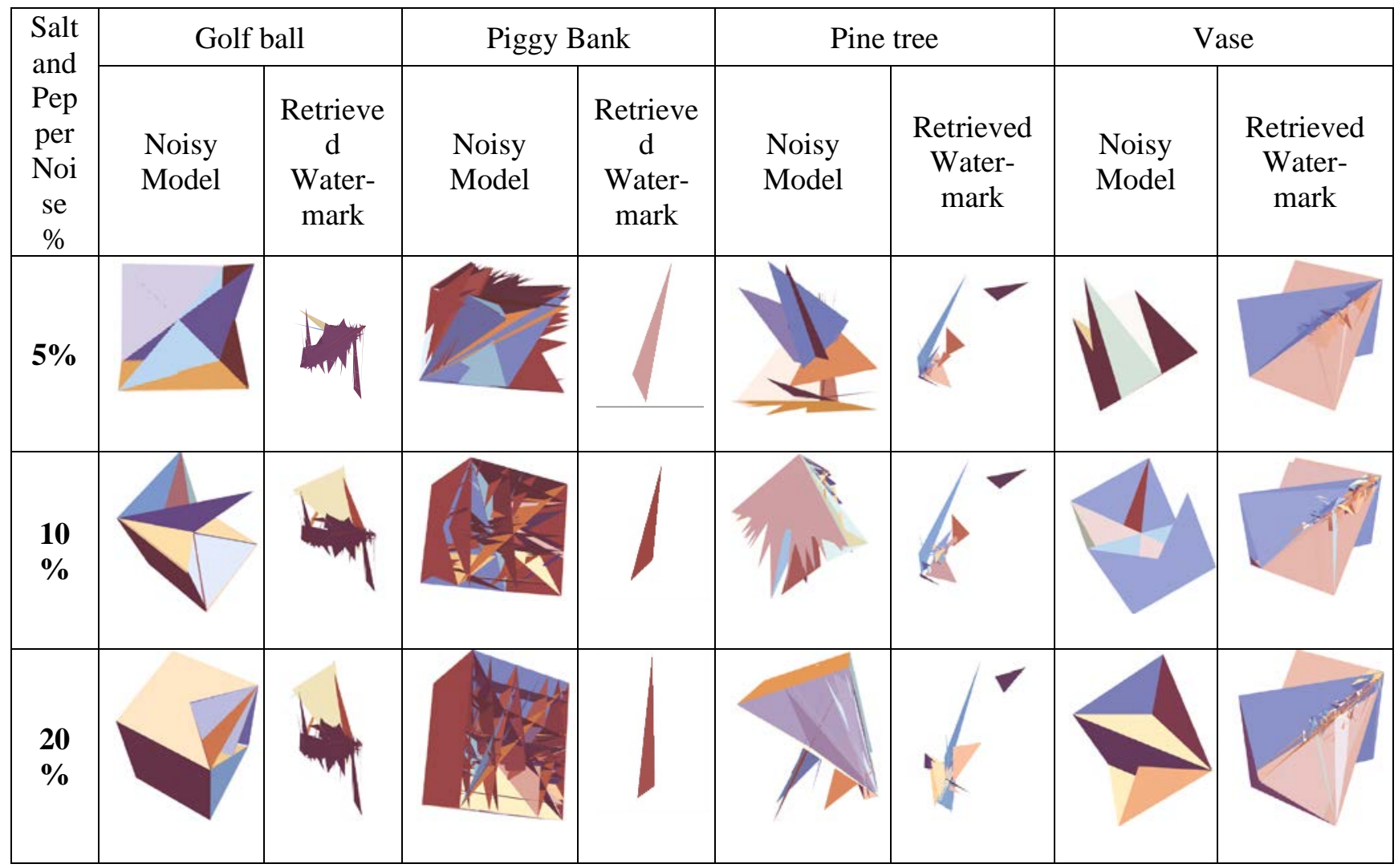

Fig. 4(a). Noisy 3D model and the extracted watermarks (Salt and Pepper)

Table 5. Types of Attacks

\begin{tabular}{|c|ccc|}
\hline Types of Attack & \multicolumn{3}{|c|}{ Characters } \\
\hline Noise (Salt and Pepper noise) & $0.5 \%$ & $1 \%$ & $2 \%$ \\
\hline Noise (Gaussian noise) & $0.5 \%$ & $1 \%$ & $2 \%$ \\
\hline Cropping & $10 \%$ & $20 \%$ & $30 \%$ \\
\hline Scaling Factors & 0.5 & 1.5 & 2 \\
Axis & $\mathrm{X}$ & $\mathrm{Y}$ & $\mathrm{Z}$ \\
\hline
\end{tabular}



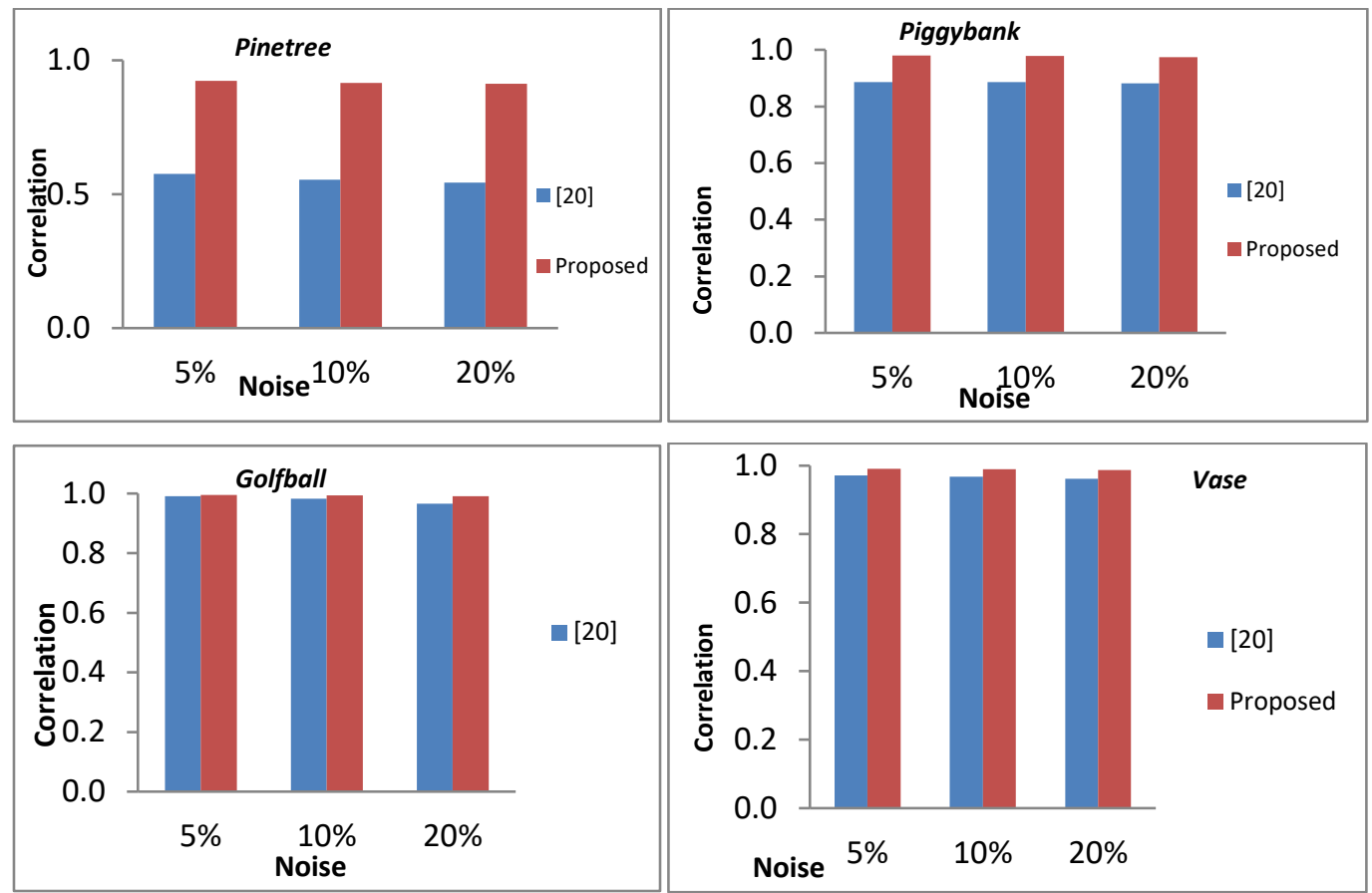

Fig. 5(a). NC values between the original retrieved 3D watermark from a salt and pepper noisy model.
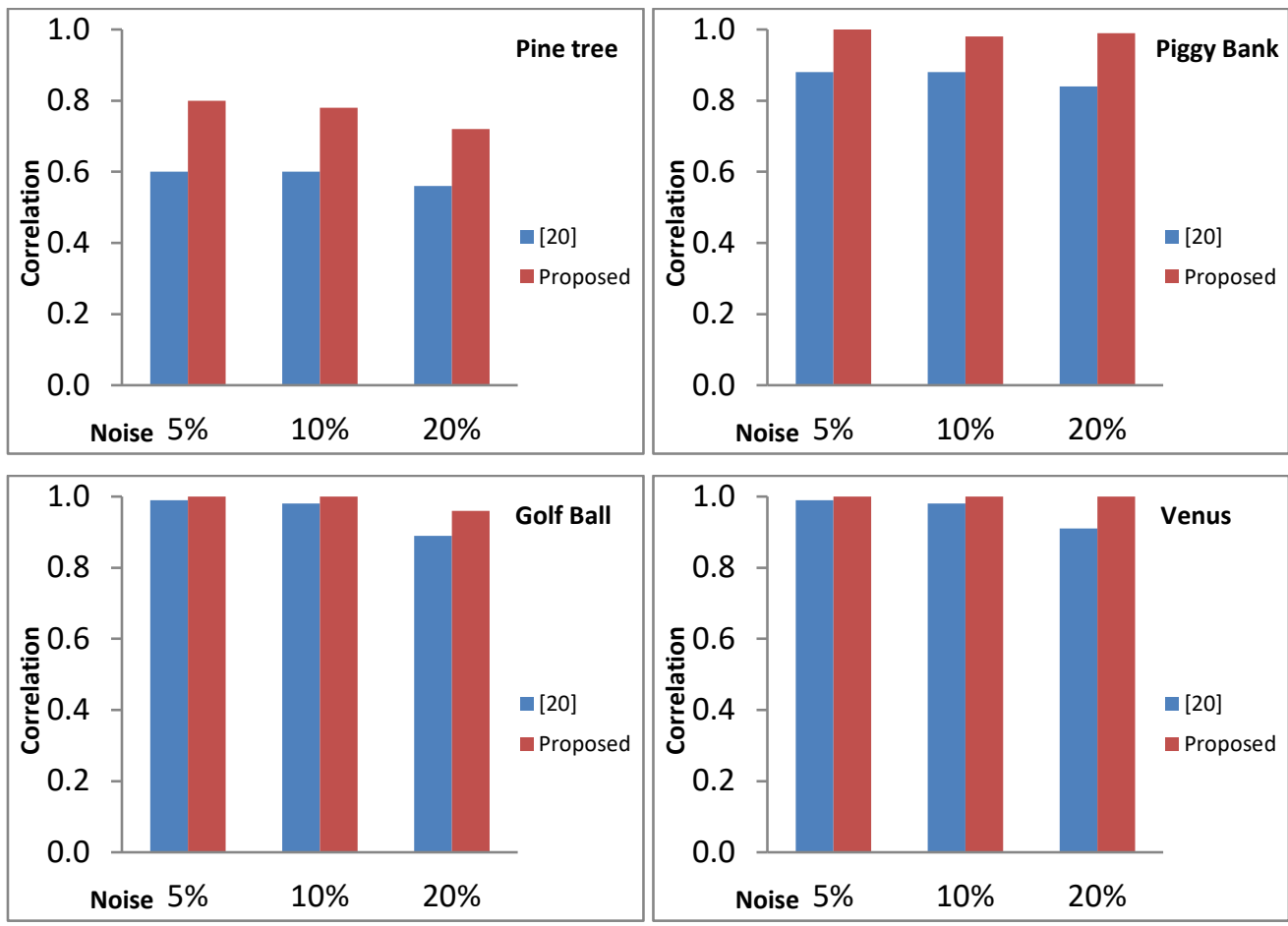

Fig. 5(b). NC values between the original retrieved 3D watermark from a Gaussian noisy model. 


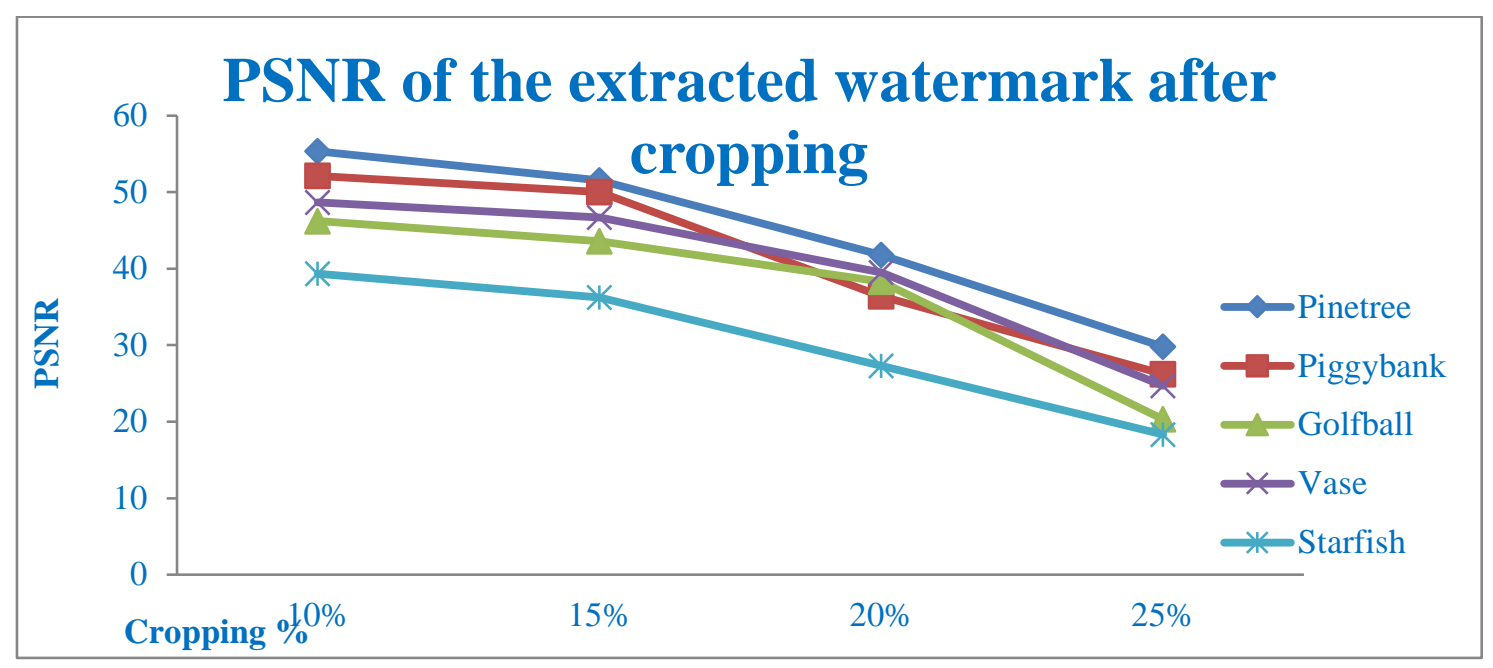

Fig. 6. PSNR of the generated watermark after cropping vertices

Table 6. Performance comparison of the proposed scheme to resist scaling attacks

\begin{tabular}{|l|c|c|}
\hline \multirow{2}{*}{ Object } & $\begin{array}{c}\text { Correlation value of Scaled Object } \\
(\mathrm{x}=0.5, \mathrm{y}=1.5, \mathrm{z}=2)\end{array}$ \\
\cline { 2 - 3 } & {$[20] \alpha=0.01$} & [proposed work] \\
\hline Pinetree & 0.1851 & 0.3522 \\
\hline Piggybank & -0.6967 & 0.5070 \\
\hline Golfball & 0.4888 & 0.6056 \\
\hline Vase & 0.2173 & 0.6789 \\
\hline
\end{tabular}

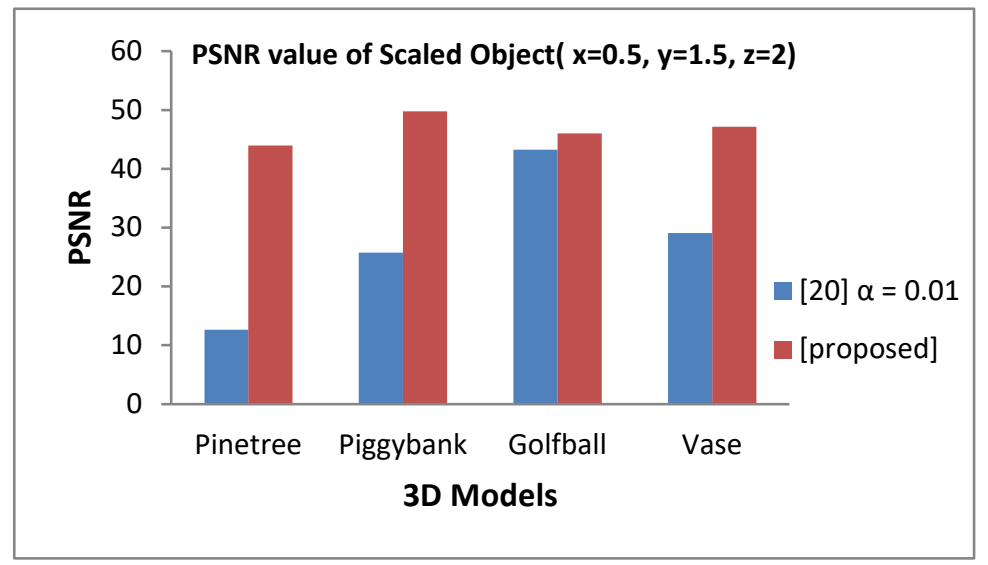

Fig. 7. Performance comparison to counter scaling attacks for the proposed model in terms of detection ratio. 


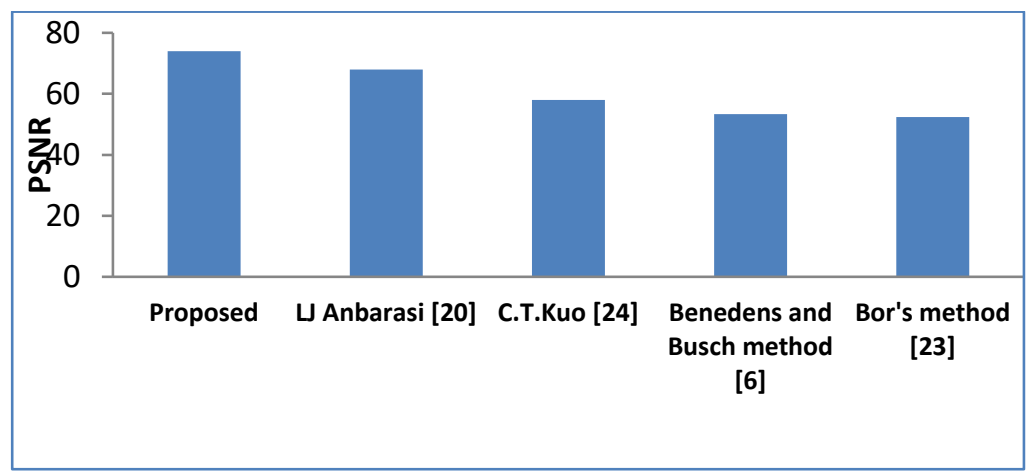

Fig. 8. PSNR accuracy obtained for the existing systems and the proposed ACO-Weber Scheme

\section{Conclusion}

An optimized reliable ACO based Weber law watermarking scheme is proposed achieving a high robustness without losing imperceptibility. ACO attains an elite strength factor which achieved a good imperceptibility target. A watermark is generated from the original 3D model using the Weber Law. The generated 3D secret watermark is embedded into the host 3D mesh model. Embedding and extraction are achieved in the wavelet domain using the optimized strength factor. The achieved experimental results show good robustness towards different attacks including additive noise addition, cropping and scaling, thus demonstrating the efficiency of the algorithm. The ACO-Weber watermarking scheme exhibits good resistance towards various geometrical and non-geometrical attacks. Going forward, further analysis is called for to exploit the watermarking technique so as to embed and extract watermarks using blind schemes.

\section{Rererences}

[1] Abdallah, Emad E., A. Ben Hamza, and Prabir Bhattacharya, "Spectral graph-theoretic approach to 3D mesh watermarking," in Proc. of graphics interface 2007. ACM, pp. 327-334, 2007. Article (CrossRef Link)

[2] Metnon, N., and P. Wong, "Protecting multimedia content," Commun. ACM, 47.7, 35-43, 1998. Article (CrossRef Link)

[3] Cox Ingemar J., Matthew L. Miller, and Jeffrey A. Bloom, "8 - Robust Watermarking,” Digital watermarking, 241-277, 2002. Article (CrossRef Link)

[4] Hartung, Frank, and Martin Kutter, "Multimedia watermarking techniques," Proceedings of the IEEE, 87.7, 1079-1107, 1999. Article (CrossRef Link)

[5] Praun, Emil, Hugues Hoppe, and Adam Finkelstein, "Robust mesh watermarking," in Proc. of Siggraph, Vol. 99, pp. 49-56, 1999. Article (CrossRef Link)

[6] Benedens, Oliver, and Christoph Busch, "Towards blind detection of robust watermarks in polygonal models," in Proc. of Computer Graphics Forum, Vol. 19, No. 3, pp. 199-208, 2000. Article (CrossRef Link)

[7] Ohbuchi, Ryutarou, et al, "Watermarking 3D polygonal meshes in the mesh spectral domain," Graphics interface, Vol. 2001, pp. 9-18, 2001. Article (CrossRef Link)

[8] Harte, Thomas, and Adrian G. Bors, "Watermarking 3D models," in Proc. of International Conference on Image Processing, Vol. 3, 2002. Article (CrossRef Link)

[9] Cotting, Daniel, et al, "Robust watermarking of point-sampled geometry," in Proc. of Shape Modeling Applications, 2004. IEEE, 2004. Article (CrossRef Link) 
[10] $\mathrm{Li}$, Li, et al, "Watermarking subdivision surfaces based on addition property of Fourier transform," in Proc. of the 2nd international conference on Computer graphics and interactive techniques in Australasia and South East Asia. ACM, pp. 46-49, 2004. Article (CrossRef Link)

[11] Jian-qiu, Jin, et al, "Watermarking on 3D mesh based on spherical wavelet transform," Journal of Zhejiang University-Science A, 5.3, 251-258, 2004. Article (CrossRef Link)

[12] Kwon, Ki-Ryong, et al, "Watermarking for 3D polygonal meshes using normal vector distributions of each patch," in Proc. of 2003 International Conference on Image Processing (Cat. No. 03CH37429), Vol. 2, 2003. Article (CrossRef Link)

[13] Wu, Jianhua, and Leif Kobbelt, "Efficient spectral watermarking of large meshes with orthogonal basis functions,” The Visual Computer, 21.8-10, 848-857, 2005. Article (CrossRef Link)

[14] Uccheddu, Francesca, Massimiliano Corsini, and Mauro Barni, "Wavelet-based blind watermarking of 3D models," in Proc. of the 2004 workshop on Multimedia and security. ACM, pp. 143-154, 2004. Article (CrossRef Link)

[15] Garcia, Emmanuel, and Jean-Luc Dugelay, "Texture-based watermarking of 3D video objects," IEEE Transactions on Circuits and Systems for Video Technology, 13.8, 853-866, 2003. Article (CrossRef Link)

[16] Zafeiriou, Stefanos, Anastasios Tefas, and Ioannis Pitas, "Blind robust watermarking schemes for copyright protection of 3D mesh objects," IEEE Transactions on Visualization and Computer Graphics, 11.5, 596-607, 2005. Article (CrossRef Link)

[17] M. Dorigo, M. Birattari and T. Stutzle, "Ant colony optimization," IEEE Computational Intelligence Magazine, vol. 1, no. 4, pp. 28-39, Nov. 2006. Article (CrossRef Link)

[18] Makbol, Nasrin M., et al, "A new reliable optimized image watermarking scheme based on the integer wavelet transform and singular value decomposition for copyright protection," Information Sciences, 417, 381-400, 2017. Article (CrossRef Link)

[19] Dorigo, Marco, and Mauro Birattari, Ant colony optimization, Springer, Boston, MA, 2017. Article (CrossRef Link)

[20] Jani Anbarasi, L., and Modigari Narendra, "Robust watermarking scheme using Weber Law for 3D mesh models,” The Imaging Science Journal, 65.7, 409-417, 2017. Article (CrossRef Link)

[21] Laouamer, Lamri, et al, "Robust watermarking scheme and tamper detection based on threshold versus intensity,” Journal of Innovation in Digital Ecosystems, 2.1-2, 1-12, 2015. Article (CrossRef Link)

[22] Karni, Zachi, and Craig Gotsman, "Spectral compression of mesh geometry," in Proc. of the 27th annual conference on Computer graphics and interactive techniques. ACM Press/AddisonWesley Publishing Co., pp. 279-286, 2000. Article (CrossRef Link)

[23] Bors, Adrian G, "Watermarking mesh-based representations of 3-D objects using local moments," IEEE Transactions on Image processing, 15.3, 687-701, 2006. Article (CrossRef Link)

[24] Kuo, Chen-Tsung, et al, "A blind robust watermarking scheme for 3D triangular mesh models using 3d edge vertex detection," Asian Journal of Health and Information Sciences, 4.1, 36-63, 2009.

[25] Cignoni, Paolo, Claudio Rocchini, and Roberto Scopigno, "Metro: Measuring error on simplified surfaces," Computer Graphics Forum, Vol. 17. No. 2, pp. 167-174, 1998. Article (CrossRef Link)

[26] Borah, S., Borah, B., "A Blind. Semi-Fragile 3D mesh Watermarking Algorithm Using Minimum Distortion Angle Quantization Index Modulation (3D-MDAQIM)," Arab J Sci Eng, 44, 3867-3882, 2019. Article (CrossRef Link)

[27] Sagarika Borah, Bhogeswar Borah, "Watermarking Techniques for Three Dimensional (3D) Mesh Authentication in Spatial Domain," 3D Research, vol. 9, eptember 2018. Article (CrossRef Link)

[28] Jang, HU., Choi, HY., Son, J. et al, "Cropping-resilient 3D mesh watermarking based on consistent segmentation and mesh steganalysis," Multimedia Tools and Applications, Vol. 77, Issue 5, pp. 5685-5712, 2018. Article (CrossRef Link) 
[29] J. U. Hou, D. G. Kim and H. K. Lee, "Blind 3D Mesh Watermarking for 3D Printed Model by Analyzing Layering Artifact," IEEE Transactions on Information Forensics and Security, vol. 12, no. 11, pp. 2712-2725, Nov. 2017. Article (CrossRef Link)

[30] Raj, Benson, et al, "A New Transformation of 3D Models Using Chaotic Encryption Based on Arnold Cat Map," in Proc. of International Conference on Emerging Internetworking, Data \& Web Technologies. Springer, Cham, pp. 322-332, 2019. Article (CrossRef Link)

[31] Socha, Krzysztof, and Marco Dorigo, "Ant colony optimization for continuous domains," European journal of operational research, 185.3, 1155-1173, 2008. Article (CrossRef Link)

[32] Ping Guo, Lin Zhu, “Ant Colony Optimization for Continuous Domains," in Proc. of 2012 8th International Conference on Natural Computation (ICNC 2012), 2012. Article (CrossRef Link)

[33] Kai Wang Guillaume Lavou'e, Florence Denis and Attila Baskurt, "Three Dimensional Meshes Watermarking: Review and Attack- Centric Investigation," in Proc. of Conference on Information Hiding 2007, pp. 50-64, March 2007. Article (CrossRef Link)

[34] Xiangui Kang, Jiwu Huang, Yun Q Shi, Yan Lin, "A DWT-DFT Composite Watermarking Scheme Robust to Both Affine Transform and JPEG Compression," IEEE Transactions on Circuits and Systems for Video Technology, Vol 13, No. 8, pp. 776-786, Aug 2003.

Article (CrossRef Link)

[35] S. Vallet and R. Prost, "Wavelet- Based Multiresolution Analysis of Irregular Surface Meshes," IEEE trans. On Visualization and Computer Graphics, vol 10, no. 2, pp. 113-122, Mar. Apr. 2004. Article (CrossRef Link)

[36] M. S. Kim, S. Vallete, H. Y. Jung and R. Prost, "Watermarking of 3D Irregular Meshes based on Wavelet Multiresolution Analysis," in Proc. of International workshop on Digital Watermarking (IWDW'05), pp. 313-324, 2005. Article (CrossRef Link)

[37] Xuefang Li, Randing Wang, "A Video Watermarking Scheme based on 3D DWT and Neural Network," in Proc. of Ninth IEEE International Symposium on Multimedia 2007- workshops, 2007. Article (CrossRef Link)

[38] Shi-Chun Mei, Ren-Hou Li, Hong-Mei Dang, Yun-Kuan, Wang, "Decision of Image Watermarking Strength based on Artificial Neural Network," in Proc. of 9th International Conference on Neural Information Processing, ICONIP' 02, vol. 5, pp. 2430-2434, 18-22 Nov. 2002. Article (CrossRef Link)

[39] Mukesh C. Motwani, Bobby D. Bryant, Sergiu M. Dascalu, and Frederick C. Harris, Jr., "3d Multimedia Protection Using Artificial Neural Network," in Proc. of IEEE CCNC 2010 proceedings, 2010. Article (CrossRef Link) 


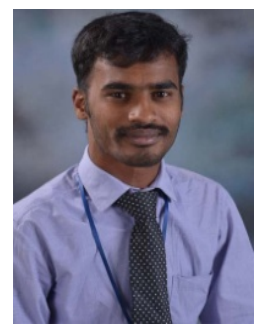

Modigari Narendra, Working as an Assistant Professor in the department of Computer Science and Engineering, Vignan's Foundation for Science Technology and Research, Guntur, India. Currently pursuing Ph.D. in the area of 3D Modeling and have expertise in algorithms, information security, networks.

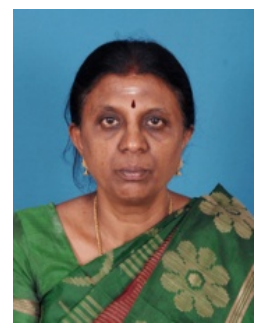

Dr. M.L.Valarmathi is working as an professor in the department of Computer Science and Engineering, Dr. Mahalingam College of Engineering and Technology, Pollachi, India. Research Expertise in the area of Solar Systems, Soft Computing, image processing, data mining, networks, 3D modeling. Authored for more than 100 research articles with 630+ citations, and i10-index of 17.

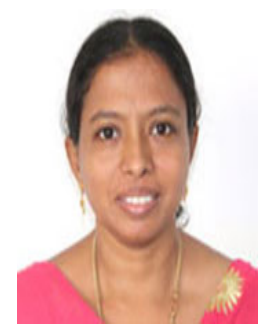

Dr. L Jani Anbarasi is working as Assistant Professor in the department of Computer Science and Engineering, Vellore Institute of Technology, Chennai. Expertise in various fields of research like Image Processing, Visual Cryptography, Cloud Computing, Big data analytics. Authored for more than 40 research articles. 